PROCEEDINGS OF THE

AMERICAN MATHEMATICAL SOCIETY

Volume 129, Number 11, Pages 3193-3200

S 0002-9939(01)05962-7

Article electronically published on May 21, 2001

\title{
CHARACTERIZATION OF COMPLETIONS OF REDUCED LOCAL RINGS
}

\author{
DAN LEE, LEANNE LEER, SHARA PILCH, AND YU YASUFUKU
}

(Communicated by Wolmer V. Vasconcelos)

\begin{abstract}
We find necessary and sufficient conditions for a complete local ring to be the completion of a reduced local ring. Explicitly, these conditions on a complete local ring $T$ with maximal ideal $\mathfrak{m}$ are (i) $\mathfrak{m}=(0)$ or $\mathfrak{m} \notin$ Ass $T$, and (ii) for all $\mathfrak{p} \in \operatorname{Ass} T$, if $r \in \mathfrak{p}$ is an integer of $T$, then $\operatorname{Ann}_{T}(r) \not \mathfrak{p}$.
\end{abstract}

\section{INTRODUCTION}

In this paper all rings are commutative with unity. Local rings are defined to be Noetherian, while quasi-local rings are not necessarily Noetherian. When we write $(R, M)$ is a quasi-local ring, we mean that $R$ is a quasi-local ring with maximal ideal $M$. In this case $\widehat{R}$ denotes the $M$-adic completion of $R$.

In 1986, Lech solved the problem of characterizing completions of local domains, proving that a complete local ring $(T, \mathfrak{m})$ is the completion of a local domain if and only if (1) $\mathfrak{m}=(0)$ or $\mathfrak{m} \notin$ Ass $T$ and (2) no nonzero integer of $T$ is a zero-divisor [6]. Heitmann, in 1993, continued this work by finding all completions of local unique factorization domains [4. To be precise, a complete local ring $T$ is the completion of a local UFD if and only if it is a field, a discrete valuation ring, or a ring of depth at least two with no nonzero integer being a zero-divisor. Following this trend, we asked the corresponding question for reduced rings: given a complete local ring, when is it the completion of a reduced local ring? In this paper we prove a theorem that answers this question.

Theorem 1. Let $(T, \mathfrak{m})$ be a complete local ring, and let $R_{0}$ be its prime subring. Then $T$ is the completion of a reduced local ring if and only if the following two properties hold:

(i) $\mathfrak{m}=(0)$ or $\mathfrak{m} \notin \operatorname{Ass} T$.

(ii) For all $\mathfrak{p} \in \operatorname{Ass} T$, if $r \in \mathfrak{p} \cap R_{0}$, then $\operatorname{Ann}_{T}(r) \nsubseteq \mathbb{p}$.

To illustrate the applicability of this theorem, we include two examples of complete local rings and ask whether they are completions of reduced local rings. In both cases, we know that they are not completions of local domains by Lech's Theorem.

Received by the editors January 18, 2000 and, in revised form, March 27, 2000.

2000 Mathematics Subject Classification. Primary 13B35.

Key words and phrases. Reduced rings, completions.

This research was supported by NSF Grant DMS-9820570 and conducted as part of the Williams College Math REU under the guidance of advisor S. Loepp.

(C)2001 American Mathematical Society 
Example 1. Let $p$ be a prime integer, and let $\widehat{\mathbb{Z}}_{(p)}$ denote the $p$-adic integers. Let $T_{1}=\widehat{\mathbb{Z}}_{(p)} \llbracket x, y \rrbracket /\left(p x, y^{2}\right)$. In this case, Ass $T_{1}=\{(x, y),(p, y)\}$, so (i) of Theorem廿 is satisfied. Moreover, the prime subring is $\mathbb{Z}$, and

$$
\begin{aligned}
& (x, y) \cap \mathbb{Z}=(0), \\
& (p, y) \cap \mathbb{Z}=p \mathbb{Z} \text { and } x p=0 \text { with } x \notin(p, y) .
\end{aligned}
$$

Thus, by Theorem $1, T_{1}$ is the completion of a reduced local ring.

Example 2. Let $T_{2}=\widehat{\mathbb{Z}}_{(p)} \llbracket x, y \rrbracket /\left(p x, x^{2}\right)$. In this case, $(p, x) \in \operatorname{Ass} T_{2}$ and the prime subring is $\mathbb{Z}$. Note that $p \in(p, x) \cap \mathbb{Z}$ and no element outside of $(p, x)$ annihilates $p$, so condition (ii) of Theorem 1 is not satisfied. So $T_{2}$ is not the completion of a reduced local ring, although the prime subring is reduced.

To prove Theorem[1, we use a construction based on that of Heitmann [4]. Specifically, we use Proposition 1 in [5] which states that if $(A, \mathfrak{m} \cap A)$ is a quasi-local subring of a complete local ring $(T, \mathfrak{m}), A \rightarrow T / \mathfrak{m}^{2}$ is surjective, and $I T \cap A=I$ for every finitely generated ideal $I$ of $A$, then $A$ is Noetherian and the natural homomorphism $\widehat{A} \rightarrow T$ is an isomorphism. We begin with the prime subring of $T$ and construct an increasing chain of rings whose union satisfies the conditions of the proposition. During this process, we construct each ring so that it is reduced. However, it is not possible to simply preserve reducedness at each step (see Example 2). We need to carry through a slightly stronger condition on each ring $R$ : for all $\mathfrak{p} \in \operatorname{Ass} T$, if $r \in \mathfrak{p} \cap R$, then $\operatorname{Ann}_{T}(r) \nsubseteq \mathfrak{p}$.

\section{Proof of Theorem 1}

Proof of necessity. Assume that $T=\widehat{A}$ for some reduced local ring $A$. Then we claim that $\mathfrak{p} \cap A$ is a minimal prime of $A$ for any $\mathfrak{p} \in \operatorname{Ass} T$. Note that $\mathfrak{p} \cap A$ is a set of elements in $A$ which are zero-divisors in $T$, so $\mathfrak{p} \cap A$ is actually a set of zero-divisors in $A$. Since $A$ is reduced, $\mathfrak{p} \cap A$ must be contained in the union of minimal primes of $A$; by prime avoidance and minimality, it follows that $\mathfrak{p} \cap A$ is a minimal prime. Now, we will check property (i). If $\mathfrak{m} \in \operatorname{Ass} T$, then by our claim, $\mathfrak{m} \cap A$ is a minimal prime of $A$. But $\mathfrak{m} \cap A$ is a maximal prime of $A$ by faithful flatness, so $\mathfrak{m} \cap A$ is the nilradical of $A$ which is $(0)$. Thus $\mathfrak{m}=(\mathfrak{m} \cap A) T=(0)$. To check (ii), let $a \in \mathfrak{p} \cap A$ for some $\mathfrak{p} \in \operatorname{Ass} T$. Since $\mathfrak{p} \cap A$ is a minimal prime of a reduced ring $A, A_{\mathfrak{p} \cap A}$ is a field. Hence, $a$ must be annihilated by an element in $A-(\mathfrak{p} \cap A)$. Since $A$ contains $R_{0}$, (ii) follows.

The remainder of this paper deals with the proof of sufficiency. We shall show that if $(T, \mathfrak{m})$ is a complete local ring with properties (i) and (ii) of Theorem 1 then we can construct a reduced local ring $A$ such that $\widehat{A}=T$. Note first that if $\mathfrak{m}=(0)$, then $T$ is a field, and so $T$ is the completion of a reduced local ring, namely itself. Thus we now prove the theorem for the case $\mathfrak{m} \notin$ Ass $T$. In order to construct a reduced local ring that completes to $T$, we will need the following proposition, which is essentially a "completion-proving machine." The proof of this proposition can be found in [5].

Proposition 2. Let $(A, \mathfrak{m} \cap A)$ be a quasi-local subring of a complete local ring $(T, \mathfrak{m})$. Assume that $A$ surjects onto $T / \mathfrak{m}^{2}$ and for every finitely generated ideal $I$ in $A, I T \cap A=I$. Then $A$ is Noetherian and the natural homomorphism $\widehat{A} \longrightarrow T$ is an isomorphism. 
Let $(T, \mathfrak{m})$ be a complete local ring, $R$ a quasi-local subring of $T$, and $C$ a subset of Spec $T$. In our construction we will want to choose elements of $T$ that are transcendental over $R /(P \cap R)$ as elements of $T / P$ for all $P \in C$. The following two lemmas allow us to do this. These lemmas and their proofs can be found in [4] as Lemma 2 and Lemma 3.

Lemma 3. Let $T$ be a complete local ring with maximal ideal $\mathfrak{m}, C$ a countable subset of $\operatorname{Spec} T$ such that $\mathfrak{m} \notin C$, and $D$ a countable set of elements of $T$. If $I$ is an ideal of $T$ which is contained in no single $P$ in $C$, then $I \nsubseteq \bigcup\{P+r \mid P \in C, r \in D\}$.

Lemma 4. Let $(T, \mathfrak{m})$ be a local ring. Let $C \subseteq \operatorname{Spec} T, D \subseteq T$, and let $I$ be an ideal of $T$ such that $I \nsubseteq P$ for all $P \in C$. Suppose $|C \times D|<|T / \mathfrak{m}|$. Then $I \nsubseteq \bigcup\{P+r \mid P \in C, r \in D\}$.

We will use the following definition in the construction of our reduced subrings.

Definition. Let $(R, \mathfrak{m} \cap R)$ be a quasi-local subring of a complete local ring $(T, \mathfrak{m})$. Then $R$ is an $\mathbf{L}$-subring of $T$ iff:

(i) If $T / \mathfrak{m}$ is countable, then $R$ is countable. Otherwise, $|R|<|T / \mathfrak{m}|$.

(ii) For all $\mathfrak{p} \in \operatorname{Ass} T$, if $r \in \mathfrak{p} \cap R$, then there exists $s \in T-\mathfrak{p}$ such that $s r=0$.

At each stage of the construction we will want our constructed intermediate subrings to be L-subrings. In the following lemma, we prove that condition (ii) of L-subrings will ensure that all of these subrings will be reduced.

Lemma 5. Let $R \subseteq T$ be rings. Assume that for all $\mathfrak{p} \in$ Ass $T$ and $r \in \mathfrak{p} \cap R$, there exists $s \in T-\mathfrak{p}$ such that $s r=0$. Then $R$ is reduced.

Proof. Suppose $r$ is a nonzero element of the nilradical of $R$. Let $n$ be the smallest integer such that $r^{n}=0$. So $n>1$ and $r^{n-1} \neq 0$. Then $r \in \operatorname{Ann}_{T}\left(r^{n-1}\right) \subseteq \mathfrak{p}$ for some $\mathfrak{p} \in \operatorname{Ass} T$. So by assumption, there exists $s \notin \mathfrak{p}$ such that $s r=0$. Then $s \in \operatorname{Ann}_{T}\left(r^{n-1}\right) \subseteq \mathfrak{p}$, which gives a contradiction.

Definition. If $R$ is an L-subring of a complete local $\operatorname{ring} T$, then $S \supseteq R$ is called a B-extension of $R$ if $S$ is also an L-subring of $T$ and $|S| \leq \max \left(\aleph_{0},|R|\right)$.

Note that the B-extension relation is transitive.

The crucial lemma of this proof is the following one. We will use it to assist us in constructing rings that satisfy the finitely generated ideal condition of Proposition 2 ,

Lemma 6. Let $R$ be an L-subring of a complete local ring $(T, \mathfrak{m})$ with $\mathfrak{m} \notin$ Ass $T$. Let $I$ be a finitely generated ideal of $R$, and let $c \in I T \cap R$. Then there exists a $B$-extension $S$ of $R$ with $c \in I S$.

Proof. We proceed by induction on $m$, the number of generators of $I$. Let $c \in I T$ $\cap R$. First consider the case $m=1$ where $I=y R$ for some $y \in R$. We first partition Ass $T=\left\{\mathfrak{p}_{1}, \ldots, \mathfrak{p}_{\mu}\right\} \cup\left\{\mathfrak{q}_{1}, \ldots, \mathfrak{q}_{\nu}\right\}$ so that $y \in \mathfrak{p}_{i}$ for all $i$ and $y \notin \mathfrak{q}_{j}$ for all $j$. Without loss of generality, take $\mathfrak{p}_{1}, \ldots, \mathfrak{p}_{k}$ to be the maximal elements of the set $\left\{\mathfrak{p}_{1}, \ldots, \mathfrak{p}_{\mu}\right\}$. Since $R$ is an L-subring, by condition (ii), for each $i \leq k$, there exists $s_{i} \notin \mathfrak{p}_{i}$ such that $s_{i} y=0$. For all $i, j \leq k$ with $i \neq j$, choose an element $r_{i j} \in \mathfrak{p}_{j}-\mathfrak{p}_{i}$. Define $u_{i}=s_{i} \prod_{j \neq i}^{k} r_{i j}$. Then $u_{i} \notin \mathfrak{p}_{i}$ and $u_{i} \in \mathfrak{p}_{j}$ for all $j \neq i$ while $u_{i}$ annihilates $y$. Now define $s=\sum_{i=1}^{k} u_{i}$. Then $s y=0$ while $s \notin \mathfrak{p}_{i}$ for all $i \leq k$, and consequently, $s \notin \mathfrak{p}_{i}$ for all $i \leq \mu$. 
Since $c \in y T, c=y t$ for some $t \in T$. Note that $c=y(t+a s)$ for any choice of $a \in T$. For each $i$, let $D_{i}$ be a full set of coset representatives for those choices of $v \in T$ which make $\overline{t+v} \in T / \mathfrak{p}_{i}$ algebraic over $R /\left(\mathfrak{p}_{i} \cap R\right)$. Let $D=\bigcup D_{i}$ and $C=\left\{\mathfrak{p}_{1}, \ldots, \mathfrak{p}_{\mu}\right\}$. Notice that $R$ satisfies condition (i) of the L-subring, $\mathfrak{m} \notin C$, and $s T \nsubseteq \mathfrak{p}_{i}$ for all $i$. Therefore we may apply Lemma 3 when $T / \mathfrak{m}$ is countable or Lemma 4 otherwise, which gives us an element as $\in s T$ such that as $\notin \bigcup\left\{\mathfrak{p}_{i}+v \mid i \leq\right.$ $\mu, v \in D\}$. So $t^{\prime}=t+a s \notin \bigcup\left\{\mathfrak{p}_{i}+(t+v) \mid i \leq \mu, v \in D\right\}$. Therefore $\overline{t^{\prime}}=\overline{t+a s} \in T / \mathfrak{p}_{i}$ is transcendental over $R /\left(\mathfrak{p}_{i} \cap R\right)$ for all $i$.

We claim that $S=R\left[t^{\prime}\right]_{\mathfrak{m} \cap R\left[t^{\prime}\right]}$ is the desired extension. It is clear that $c \in I S$, $R \subseteq S \subseteq T, S$ is quasi-local, and that $|S| \leq \max \left(\aleph_{0},|R|\right)$. Therefore we only need to check condition (ii) of L-subrings. It suffices to check condition (ii) on $R\left[t^{\prime}\right]$. Pick $\mathfrak{P} \in \operatorname{Ass} T$.

Case $1: \mathfrak{P}=\mathfrak{p}_{i}$ for some $i$.

Let $f \in \mathfrak{p}_{i} \cap R\left[t^{\prime}\right]$. Write $f=r_{n}\left(t^{\prime}\right)^{n}+\cdots+r_{0}$ for some $r_{j} \in R$. Since $\overline{t^{\prime}} \in T / \mathfrak{p}_{i}$ is transcendental over $R /\left(\mathfrak{p}_{i} \cap R\right)$, we see that each $r_{j} \in \mathfrak{p}_{i} \cap R$. By condition (ii) on $R$, for each $j$, there exists $b_{j} \notin \mathfrak{p}_{i}$ such that $b_{j} r_{j}=0$. Thus $\prod_{j=1}^{n} b_{j}$ annihilates $f$ but is not contained in $\mathfrak{p}_{i}$.

Case $2: \mathfrak{P}=\mathfrak{q}_{i}$ for some $i$.

Let $f \in \mathfrak{q}_{i} \cap R\left[t^{\prime}\right]$. Writing $f$ as before, we see that $y^{n} f \in R$. So $y^{n} f \in \mathfrak{q}_{i} \cap R$. By condition (ii), there exists $b \notin \mathfrak{q}_{i}$ such that $b y^{n} f=0$. Notice $b y^{n} \notin \mathfrak{q}_{i}$.

This completes the $m=1$ step of the induction.

Now let $m>1$. So $I=\left(y_{1}, \ldots, y_{m}\right) R$ for some $y_{1}, \ldots, y_{m} \in R$. Define $J=\left(y_{1}, \ldots, y_{m-1}\right) R$. We know $c=t_{1} y_{1}+\cdots+t_{m} y_{m}$ for some $t_{1}, \ldots, t_{m} \in T$. Just as in the $m=1$ case, we choose $s \in \operatorname{Ann}_{T}\left(y_{m}\right)$ such that $s \notin \mathfrak{p}$ for all $\mathfrak{p} \in$ Ass $T$ containing $y_{m}$. Define $t^{\prime}=t_{m}+a_{0} s+a_{1} y_{1}+\cdots+a_{m-1} y_{m-1}$ where the $a_{i}$ 's are to be chosen later. Observe that $c=t^{\prime} y_{m}+t_{1}^{\prime} y_{1}+\cdots+t_{m-1}^{\prime} y_{m-1}$ where $t_{i}^{\prime}=t_{i}-a_{i} y_{m}$.

For all $0<i<m$, define $C_{i}=\left\{\mathfrak{p} \in \operatorname{Ass} T \mid y_{i} \notin \mathfrak{p}\right\}$. Also let $C_{0}=\{\mathfrak{p} \in \operatorname{Ass} T \mid s \notin \mathfrak{p}\}$ $=\left\{\mathfrak{p} \in \operatorname{Ass} T \mid y_{m} \in \mathfrak{p}\right\}$. Finally, define $C=C_{0} \cup C_{1} \cup \cdots \cup C_{m-1}$. As in the $m=1$ case, we can use Lemmas 3 and 4 to choose $a_{0}$ such that $\overline{t_{m}+a_{0} s} \in T / \mathfrak{p}$ is transcendental over $R /(\mathfrak{p} \cap R)$ for all $\mathfrak{p} \in C_{0}$. Once this is done, we can choose $a_{1}$ such that $\overline{t_{m}+a_{0} s+a_{1} y_{1}} \in T / \mathfrak{p}$ is transcendental over $R /(\mathfrak{p} \cap R)$ for all $\mathfrak{p} \in C_{1}$. We continue this process all the way to $a_{m-1}$. Observe that with these choices, $\overline{t^{\prime}} \in T / \mathfrak{p}$ is transcendental over $R /(\mathfrak{p} \cap R)$ for all $\mathfrak{p} \in C$. We claim that $R^{\prime}=R\left[t^{\prime}\right]_{\mathfrak{m} \cap R\left[t^{\prime}\right]}$ is a B-extension of $R$. $R^{\prime}$ is clearly quasi-local and satisfies $\left|R^{\prime}\right| \leq \max \left(\aleph_{0},|R|\right)$. Again, it suffices to check condition (ii) on $R\left[t^{\prime}\right]$.

Let $\mathfrak{p} \in \operatorname{Ass} T$.

Case $1: \mathfrak{p} \in C$.

Let $f \in \mathfrak{p} \cap R\left[t^{\prime}\right]$. The proof is the same as Case 1 when $m=1$.

Case $2: \mathfrak{p} \notin C$.

This means that $y_{m} \notin \mathfrak{p}$ while $y_{1}, \ldots, y_{m-1} \in \mathfrak{p}$. So $J T \subseteq \mathfrak{p}$. Let

$f \in \mathfrak{p} \cap R\left[t^{\prime}\right]$. Define $\xi_{1}=-\left(t_{1}^{\prime} y_{1}+\cdots+t_{m-1}^{\prime} y_{m-1}\right) \in J \bar{T}$. Then $y_{m} t^{\prime}=c+\xi_{1}$. Therefore for any $j$, defining

$$
\xi_{j}=\sum_{k=1}^{j}\left(\begin{array}{l}
j \\
k
\end{array}\right) c^{j-k} \xi_{1}^{k},
$$


we have that $\xi_{j} \in J T$ and $\left(y_{m} t^{\prime}\right)^{j}=c^{j}+\xi_{j}$. So if $f=r_{n}\left(t^{\prime}\right)^{n}+$ $r_{n-1}\left(t^{\prime}\right)^{n-1}+\cdots+r_{0}$, then

$$
\begin{aligned}
y_{m}^{n} f= & r_{n}\left(y_{m} t^{\prime}\right)^{n}+r_{n-1} y_{m}\left(y_{m} t^{\prime}\right)^{n-1}+\cdots+r_{0} y_{m}^{n} \\
= & \left(r_{n} c^{n}+r_{n-1} y_{m} c^{n-1}+\cdots+r_{0} y_{m}^{n}\right) \\
& \quad+\left(r_{n} \xi_{n}+r_{n-1} y_{m} \xi_{n-1}+\cdots+r_{1} y_{m}^{n-1} \xi_{1}\right) .
\end{aligned}
$$

Setting

$$
\xi=r_{n} \xi_{n}+r_{n-1} y_{m} \xi_{n-1}+\cdots+r_{1} y_{m}^{n-1} \xi_{1} \in J T,
$$

we get $y_{m}^{n} f-\xi \in R$ since $c \in R$. Then since $f \in \mathfrak{p}$ and $J T \subseteq \mathfrak{p}$, $y_{m}^{n} f-\xi \in \mathfrak{p} \cap R$. So by condition (ii) of L-subrings, there exists $u \in T-\mathfrak{p}$ such that $u\left(y_{m}^{n} f-\xi\right)=0$. Meanwhile, for each $i<m$, $y_{i} \in \mathfrak{p} \cap R$, there exists $u_{i} \in T-\mathfrak{p}$ such that $u_{i} y_{i}=0$. Then

$$
\left(\prod_{i<m} u_{i}\right) u y_{m}^{n} f=\left(\prod_{i<m} u_{i}\right) u \xi=0
$$

since $\xi \in\left(y_{1}, \ldots, y_{m-1}\right) T$. Notice that $y_{m}^{n}\left(\prod_{i<m} u_{i}\right) u \notin \mathfrak{p}$, satisfying condition (ii).

This completes the proof that $R^{\prime}$ is a B-extension of $R$.

Now consider $c^{\prime}=c-t^{\prime} y_{m}$. Recall that $c=t^{\prime} y_{m}+t_{1}^{\prime} y_{1}+\cdots+t_{m-1}^{\prime} y_{m-1}$ so that $c^{\prime} \in J T \cap R\left[t^{\prime}\right]$. Since $J R^{\prime}$ is generated by $m-1$ elements in $R^{\prime}$, which is an L-subring, our induction hypothesis shows that there exists a B-extension $S$ of $R^{\prime}$ with $c^{\prime} \in J S$. Then $c \in I S$, so $S$ is the desired ring.

The following lemma helps us satisfy the surjectivity condition of Proposition 2

Lemma 7. Let $R$ be an L-subring of a complete local ring $(T, \mathfrak{m})$ with $\mathfrak{m} \notin \operatorname{Ass} T$, and let $u \in T$. Then there exists a B-extension $S$ of $R$ and an element $v \in S$ such that $u-v \in \mathfrak{m}^{2}$.

Proof. Because $\mathfrak{m} \notin$ Ass $T, \mathfrak{m}^{2}$ is not contained in any associated prime. For each $\mathfrak{p} \in \operatorname{Ass} T$, let $D_{\mathfrak{p}}$ be a full set of coset representatives for those choices of $t$ which make $\overline{u+t} \in T / \mathfrak{p}$ algebraic over $R /(\mathfrak{p} \cap R)$. Let $D=\bigcup_{\mathfrak{p} \in \operatorname{Ass} T} D_{\mathfrak{p}}$. As before, we use Lemma 3 when $T / \mathfrak{m}$ is countable or Lemma 4 otherwise to obtain $t \in \mathfrak{m}^{2}$ such that $\overline{u+t} \in T / \mathfrak{p}$ is transcendental over $R /(\mathfrak{p} \cap R)$ for all $\mathfrak{p} \in \operatorname{Ass} T$. Let $v=u+t$

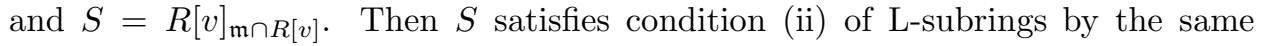
argument as in Case 1 of the $m=1$ case of the proof of Lemma 6 . The cardinality condition $|S| \leq \max \left(\aleph_{0},|R|\right)$ is obvious.

Definition. For $\Omega$ a well-ordered set and $\alpha \in \Omega$, define $\gamma(\alpha)=\sup \{\beta \in \Omega \mid \beta<\alpha\}$.

The next lemma shows that condition (ii) of L-subrings is preserved when we take the union of a chain of B-extensions.

Lemma 8. Let $\Omega$ be a well-ordered set with least element $\alpha_{0},(T, \mathfrak{m})$ a complete local ring, and $R_{\alpha_{0}}$ an L-subring of T. Suppose $\left\{R_{\alpha}\right\}_{\alpha \in \Omega}$ is an increasing chain of $L$-subrings such that if $\gamma(\alpha)<\alpha$, then $R_{\alpha}$ is a B-extension of $R_{\gamma(\alpha)}$, and otherwise $R_{\alpha}=\bigcup_{\beta<\alpha} R_{\beta}$. Then $S=\bigcup_{\alpha \in \Omega} R_{\alpha}$ satisfies condition (ii) of L-subrings, and also $|S| \leq \max \left(\aleph_{0},\left|R_{\alpha_{0}}\right|,|\Omega|\right)$. 
Proof. If $\mathfrak{p} \in \operatorname{Ass} T$ and $r \in \mathfrak{p} \cap S$, then $r \in \mathfrak{p} \cap R_{\alpha}$ for some $\alpha \in \Omega$. Then since each $R_{\alpha}$ is an L-subring, it is clear that condition (ii) of L-subrings holds for $S$. We prove by transfinite induction that for all $\alpha \in \Omega$,

$$
\left|R_{\alpha}\right| \leq \max \left(\aleph_{0},\left|R_{\alpha_{0}}\right|,|\Omega|\right)
$$

Assume that $\left|R_{\beta}\right| \leq \max \left(\aleph_{0},\left|R_{\alpha_{0}}\right|,|\Omega|\right)$ has been proved for all $\beta<\alpha$. If $\gamma(\alpha)<\alpha$, then the inequality is clear by the induction hypothesis. Otherwise, we have

$$
\begin{aligned}
\left|R_{\alpha}\right| & =\left|\bigcup_{\beta<\alpha} R_{\beta}\right| \\
& \leq \sum_{\beta<\alpha}\left|R_{\beta}\right| \\
& \leq|\{\beta \in \Omega \mid \beta<\alpha\}| \cdot \max \left(\aleph_{0},\left|R_{\alpha_{0}}\right|,|\Omega|\right) \quad \text { by the induction hypothesis } \\
& \leq \max \left(\aleph_{0},\left|R_{\alpha_{0}}\right|,|\Omega|\right) .
\end{aligned}
$$

Using this,

$$
|S| \leq|\Omega| \cdot \max \left(\aleph_{0},\left|R_{\alpha_{0}}\right|,|\Omega|\right)=\max \left(\aleph_{0},\left|R_{\alpha_{0}}\right|,|\Omega|\right)
$$

Lemma 9. Let $R$ be an L-subring of a complete local ring $(T, \mathfrak{m})$ with $\mathfrak{m} \notin$ Ass $T$, and let $u \in T$. Then there exists a B-extension $S$ of $R$ such that

(1) $S$ contains an element $v$ of $T$ such that $\bar{v}=\bar{u} \in T / \mathfrak{m}^{2}$.

(2) For every finitely generated ideal $I$ of $S, I T \cap S=I$.

Proof. By Lemma 7, there exists a B-extension $S^{(0)}$ of $R$ which contains some $v$ with $\bar{v}=\bar{u} \in T / \mathfrak{m}^{2}$. Let

$$
\Omega=\left\{(I, c) \mid I \text { finitely generated ideal of } S^{(0)} \text { and } c \in I T \cap S^{(0)}\right\} .
$$

Note that $|\Omega| \leq \max \left(\aleph_{0},\left|S^{(0)}\right|\right)=\max \left(\aleph_{0},|R|\right)$. When $\Omega$ is finite, by transitivity of the B-extension relation, we can simply apply Lemma $6|\Omega|$ times to construct a B-extension $S^{(1)}$ of $S^{(0)}$ with $I T \cap S^{(0)} \subseteq I S^{(1)}$ for any finitely generated ideal $I$ of $S^{(0)}$. In the case when $\Omega$ is infinite, we well-order $\Omega$ in such a way that there is no maximal element. Let $\alpha_{0}$ be the initial element. We shall inductively define the family of L-subrings $\left\{S_{\alpha}\right\}_{\alpha \in \Omega}$, starting from $S_{\alpha_{0}}=S^{(0)}$. Let us assume that $S_{\beta}$ has been defined for all $\beta<\alpha$. If $\gamma(\alpha) \neq \alpha$ and $\gamma(\alpha)=(I, c)$, we use Lemma 6] to define $S_{\alpha}$ to be a B-extension of $S_{\gamma(\alpha)}$ such that $c \in I S_{\alpha}$. If $\gamma(\alpha)=\alpha$, define $S_{\alpha}=\bigcup_{\beta<\alpha} S_{\beta}$; by Lemma 8, this is an L-subring. So by induction, $\left\{S_{\alpha}\right\}_{\alpha \in \Omega}$ is a family satisfying the hypotheses of Lemma 8 Now, define $S^{(1)}=\bigcup_{\alpha \in \Omega} S_{\alpha}$. By Lemma 8, this is a B-extension of $S^{(0)}$. Now let $I$ be a finitely generated ideal of $S^{(0)}$ and let $c \in I T \cap S^{(0)}$. If $\alpha$ is the successor of $(I, c)$ in $\Omega$, then we have $(I, c)=\gamma(\alpha)<\alpha$. So by construction, $c \in I S_{\alpha} \subseteq I S^{(1)}$. Thus $I T \cap S^{(0)} \subseteq I S^{(1)}$ for any finitely generated ideal $I$ of $S^{(0)}$.

We repeat this process for each $m \in \mathbb{N}$ to obtain B-extensions $S^{(m+1)}$ of $S^{(m)}$ such that for every finitely generated ideal $I$ of $S^{(m)}, I T \cap S^{(m)} \subseteq I S^{(m+1)}$. Define $S=\bigcup_{n=0}^{\infty} S^{(n)}$. By Lemma $8, S$ is a B-extension of $S^{(0)}$, so by transitivity, $S$ is a B-extension of $R$. Let $I=\left(z_{1}, \ldots, z_{k}\right)$ be a finitely generated ideal of $S$, and let 
$c \in I T \cap S$. Then there exists $n$ such that $c$ and all $z_{i}$ belong to $S^{(n)}$. Then by construction,

$$
c \in\left(\left(z_{1}, \ldots, z_{k}\right) S^{(n)}\right) T \cap S^{(n)} \subseteq\left(z_{1}, \ldots, z_{k}\right) S^{(n+1)} \subseteq I S=I .
$$

Hence, we have $I T \cap S=I$ for any finitely generated ideal $I$ of $S$.

We now have enough tools to prove sufficiency of the conditions in Theorem 1

Theorem 10. Let $(T, \mathfrak{m})$ be a complete local ring with $\mathfrak{m} \notin$ Ass $T$, and let $R_{0}$ be the prime subring of $T$. Assume that for all $\mathfrak{p} \in \operatorname{Ass} T, r \in \mathfrak{p} \cap R_{0}$ implies $\operatorname{Ann}_{T}(r) \nsubseteq \mathfrak{p}$. Then $T$ is the completion of a reduced local ring.

Proof. Note that our condition on $R_{0}$ implies that $R_{0}$ localized at $\mathfrak{m} \cap R_{0}$ is an L-subring. Call this localized ring $S_{0}$.

Claim. $\left|T / \mathfrak{m}^{2}\right| \leq|T / \mathfrak{m}|^{N+1}$ where $N$ is the number of generators of $\mathfrak{m}$.

Proof. $\mathfrak{m} / \mathfrak{m}^{2}$ is generated over $T / \mathfrak{m}$ by $N$ elements. So $\left|\mathfrak{m} / \mathfrak{m}^{2}\right| \leq$ $|T / \mathfrak{m}|^{N}$. Because $\frac{T / \mathfrak{m}^{2}}{\mathfrak{m} / \mathfrak{m}^{2}} \cong T / \mathfrak{m}$, it follows that $\left|T / \mathfrak{m}^{2}\right|=\left|\mathfrak{m} / \mathfrak{m}^{2}\right| \cdot|T / \mathfrak{m}|$ $\leq|T / \mathfrak{m}|^{N+1}$.

When $\left|T / \mathfrak{m}^{2}\right|$ is finite, we can simply apply Lemma $9\left|T / \mathfrak{m}^{2}\right|$ times to obtain an L-subring $A$ satisfying the conditions of Proposition 2, completing the proof. When $T / \mathfrak{m}^{2}$ is infinite, then the claim above shows that $|T / \mathfrak{m}|=\left|T / \mathfrak{m}^{2}\right|$. In this case, let $\Omega$ be a full set of coset representatives of $T / \mathrm{m}^{2}$, and well-order it in such a way that 0 is the initial element and every element has strictly fewer than $|\Omega|=|T / \mathfrak{m}|$ predecessors. We shall define a family of L-subrings $\left\{S_{\alpha}\right\}_{\alpha \in \Omega}$ inductively, starting from $S_{0}$. Assume that $S_{\beta}$ has been defined for all $\beta<\alpha$. If $\gamma(\alpha) \neq \alpha$, define $S_{\alpha}$ to be the B-extension of $S_{\gamma(\alpha)}$ we obtain by applying Lemma 9 , using $S_{\gamma(\alpha)}$ and the coset represented by $\gamma(\alpha)$. If $\gamma(\alpha)=\alpha$, define $S_{\alpha}=\bigcup_{\beta<\alpha} S_{\beta}$. By the way $\Omega$ is ordered and by Lemma $8, S_{\alpha}$ is an L-subring. Now define $A=\bigcup_{\alpha \in \Omega} S_{\alpha}$. This is not necessarily an L-subring, but it still satisfies property (ii), so by Lemma 5 $A$ is reduced. By the way we ordered $\Omega$, every element of $\Omega$ appears as $\gamma(\alpha)<\alpha$ for some $\alpha \in \Omega$, so it follows that the natural map $A \longrightarrow T / \mathfrak{m}^{2}$ is surjective. Moreover, by a similar argument as in the proof of Lemma 9, $I T \cap A=I$ for any finitely generated ideal $I$ of $A$. So $A$ satisfies all the hypotheses of Proposition 2 Thus we conclude that $A$ is a reduced local ring which completes to $T$.

\section{ACKNOWLEDGEMENTS}

We would like to thank S. Loepp for many helpful conversations, suggestions, and daily encouragement.

\section{REFERENCES}

1. M. F. Atiyah and I. G. Macdonald, Introduction to commutative algebra, Addison-Wesley, 1969. MR 39:4129

2. N. Bourbaki, Commutative algebra, Hermann, 1972. MR 50:12997

3. D. Eisenbud, Commutative algebra with a view toward algebraic geometry, Springer-Verlag, 1995. MR 97a:13001

4. R. Heitmann, Characterization of completions of unique factorization domains, Trans. Amer. Math. Soc. 337 (1993), 379-387. MR 93g:13006

5. Completions of local rings with an isolated singularity, J. Algebra 163 (1994), 538-567. MR 95f: 13032 
6. C. Lech, A method for constructing bad noetherian local rings, Lecture Notes in Math $\mathbf{1 1 8 3}$ (1986), 241-247. MR 87m:13010a

7. H. Matsumura, Commutative ring theory, Cambridge studies in advanced mathematics, no. 8, Cambridge University Press, 1986. MR 88h:13001

Department of Mathematics, Stanford University, Bullding 380, Stanford, CaliforNIA $94305-2125$

E-mail address: dalee@post.harvard.edu

Department of Mathematics, P.O. Box 400137, University of Virginia, CharlottesVILLE, VIRGINIA 22904-4137

E-mail address: lcl9u@virginia.edu

P.O. Box 372, WebB, Mississippi 38966

E-mail address: spilch@wso.williams.edu

Department of Mathematics, Mit, 77 Massachusetts Ave., Cambridge, Massachusetts 02139

E-mail address: yasufuku@post.harvard.edu 